\title{
¿Hacia la Ubersidad? Conflictos y contradicciones de la universidad digital
}

\section{Towards Ubersity? Conflicts and contradictions of the digital university}

\author{
Jordi Adell Segura \\ Universitat Jaume I, UJI (España) \\ Linda Castañeda Quintero \\ Universidad de Murcia, UM (España) \\ Francesc M. Esteve Mon \\ Universitat Jaume I, UJI (España)
}

\section{Resumen}

La universidad es una institución con una larga historia, que ha propiciado y sobrevivido a múltiples cambios a lo largo de los siglos. En las últimas décadas, los cambios parecen acelerarse, ligados en parte a la tecnología. La digitalización está teniendo un gran impacto en cómo desarrolla sus funciones la universidad. Pero dichos procesos no son lineales ni simples, sino que se traducen en cambios complejos, ambivalentes y conflictivos. En este trabajo se pretende analizar algunos de los efectos más relevantes de la implementación de las tecnologías digitales en la docencia e investigación universitarias desde un punto de vista sociomaterial. Es decir, no nos centraremos en esas tecnologías como objetos, ni en la realidad de las personas en ese contexto, sino en cómo las relaciones establecidas entre sujetos y objetos afectan a la acción que tiene lugar en la universidad, como realidad compleja. Para ello, el presente artículo plantea un análisis que va de lo particular a lo general. En primer lugar, se tratará brevemente cómo afecta "lo digital" a la docencia y la investigación. A continuación, se esbozarán algunas ideas sobre futuros posibles ligados a la tecnología digital y, para finalizar, se propondrán algunas ideas sobre cómo analizar el impacto de la tecnología en la universidad desde una perspectiva crítica.

Palabras clave: universidad; tecnologías de la información y de la comunicación; cambio tecnológico; innovación pedagógica.

\begin{abstract}
The university is an institution with a long history, which has fostered and survived multiple changes over the centuries. In recent decades, changes seem to be accelerating, partly linked to technology development. Digitisation is having a significant impact on how
\end{abstract}


university develops its functions, but these processes are not linear or straightforward and will translate into complex, ambivalent and conflicting changes. This paper aims to analyse some of the most relevant effects of the implementation of digital technologies in the university teaching and research, from a socio-material point of view. In other words, we will not focus on these technologies as objects, neither on people on this context, but rather on how the relationships established between subjects and objects affect the action that takes place in the university, as a complex reality. To do this, the present article presents an analysis ranging from the particular to the general. Firstly, it analyses how "the digital" affects to teaching and research, then It will sketch some ideas about possible futures linked to digital technology and, finally, will propose some ideas on how to analyse the impact of technology in the university from a critical perspective.

Keywords: university; information and communication technologies; technological change; educational innovation.

La universidad es una institución con una larga historia. A lo largo de los siglos ha sobrevivido a múltiples vicisitudes, adaptándose a las condiciones de su entorno. Sin embargo, la idea de la educación superior, sus funciones, destinatarios, estructura, etc., ha cambiado sustancialmente con el contexto, en distintos sistemas políticos y momentos históricos.

En las últimas décadas los cambios parecen haberse acelerado: la masificación, la globalización (o internacionalización), la generalización del modelo neoliberal de universidad $\mathrm{y}$, posteriormente, la transformación de la sociedad industrial en la sociedad de la información, han transformado radicalmente tanto los fines de la institución, considerada ahora un factor clave para el desarrollo económico y la competitividad global de cada país, como los medios para lograrlos, entre los que las tecnologías digitales de la información y la comunicación ocupan un lugar preeminente. Entendemos “digitalización” en sentido amplio, como la reorganización de estructuras y relaciones sociales, culturales y económicas motivada por la generalización de las tecnologías digitales de la información y la comunicación en todos los ámbitos de la actividad humana en el marco de la sociedad actual. Pero el impacto no es solo a nivel macro. A nivel personal, la revolución digital "afecta a nuestro sentido del yo, cómo nos relacionamos con los demás y cómo damos forma e interactuamos con nuestro mundo" (Floridi, 2017, p. 8).

La universidad actual está buscando su sitio en la sociedad de la información. Sin embargo, como es de esperar en una institución en la que el debate es parte fundamental de su razón de ser, la forma en que la digitalización afecta a la universidad no se manifiesta de forma lineal ni asume una única naturaleza, la "universidad digital", sino que se traduce en cambios con múltiples facetas que, en muchos casos, son difícilmente clasificables como enteramente positivos o negativos. Cambios ante los que la institución, en muchos casos, no ha asumido una posición estratégica clara o, de haberla asumido, solo ha tenido un perfil gerencial o de imagen, muy alejado 
de posiciones comprometidas con lo que creemos que son los fines propios de la universidad en nuestro tiempo.

Siendo conscientes de la impresionante cantidad de cambios que supone la introducción de las tecnologías digitales en la universidad y la actividad de las personas en ese contexto, no nos centraremos en tratarlas como meros objetos, ni en cómo ha cambiado la composición social de la universidad. En este trabajo intentaremos sugerir una aproximación a la universidad digital desde un punto de vista socio-material (Fenwick y Edwards, 2017), es decir, nos centrarnos en cuestiones sobre cómo las relaciones entre sujetos y objetos (entendidas como consustanciales e inseparables) afectan a la acción que tiene lugar en la universidad como realidad compleja. Las tecnologías educativas, por otra parte, solo alcanzan definición, funcionalidad y valor en los modelos pedagógicos que promueven, las formas de relación social que construyen y los objetivos educativos que pretenden conseguir (Hamilton y Feenberg, 2005). El impacto de la tecnología debe analizarse conjuntamente con los principios, procesos y parámetros de diseño, implementación, despliegue y uso que están en su base y que son fruto de decisiones humanas, en ocasiones impuestas, en ocasiones libremente adoptadas por los órganos de gobierno de las universidades. Un análisis crítico de la tecnología educativa en la universidad exige no descontextualizarla del trasfondo de valores, estructuras y actores que influyen en la toma de decisiones y el gobierno de nuestra sociedad.

El objetivo de este texto es sugerir algunas ideas sobre cómo analizar los efectos más relevantes de la implementación de las tecnologías digitales en la universidad, un análisis que permita proponer alternativas a sus usos habituales y afrontar el futuro de manera proactiva y no solo reactiva, es decir, con una visión. Para ello iremos de lo particular a lo general: trataremos brevemente cómo afecta "lo digital" a la docencia y la investigación, esbozaremos algunas ideas sobre futuros posibles ligados a la tecnología digital y propondremos algunas ideas sobre cómo analizar el impacto de la tecnología en la universidad desde una perspectiva crítica. Por cuestiones de espacio no vamos a tratar aspectos relacionados con la incorporación de las tecnologías digitales al New Public Management, hegemónico en la gestión universitaria, o sobre si la Digital Era Governance (Dunleavy, Margetts, Bastow y Tinkler, 2006) representa realmente una nueva manera de gestionar lo público.

Aunque la presentación de los efectos de la tecnología digital en la universidad se realice en diferentes apartados, es importante remarcar que dicha división es impostada y que, en la realidad cotidiana de la universidad, como en cualquier práctica socio-material, todas ellas se condicionan profundamente unas a otras. 


\section{LO DIGITAL EN LA UNIVERSIDAD “DE CERCA”}

\section{Conflictos de la docencia en la universidad digital}

En contra de uno de los lugares comunes que se suele utilizar cuando criticamos la docencia actual, no es cierto que si un profesor del siglo XIX (ni siquiera de la primera mitad del siglo XX) apareciera mágicamente en un aula universitaria de esta segunda década del siglo XXI, podría reconocer el contexto. El cambio tecnológico en términos materiales y funcionales ha sido espectacular. Pensemos en pizarras donde se proyectan imágenes en movimiento en tiempo real, videoconferencias, alumnos con tabletas y textos digitales, puertas electrónicas, campus virtuales con apuntes, libros, artículos, presentaciones y vídeos, wearables, redes inalámbricas, etc. Del mismo modo, las personas que estudian y trabajan en ella son ahora muy diferentes: su procedencia, inquietudes y expectativas, el papel de la mujer, las relaciones entre sexos, el lenguaje y los roles asumidos, las expectativas de futuro, etc. Tanto la perspectiva material-funcional como la perspectiva social (o de las personas) de las aulas universitarias han cambiado radicalmente.

Hay una larga tradición de literatura que se ha ocupado de forma sistemática de analizar cómo afecta la introducción de tecnologías a los procesos de enseñanza y aprendizaje, especialmente todo lo relacionado con la enseñanza a distancia, el e-learning y conceptos asociados (Area, San Nicolás y Fariña, 2010; Cabero, 2005; Martínez y Prendes, 2003; Salinas, 1999). Ahora bien, por una cuestión de espacio es imposible remontarnos a las relaciones referidas a la generalidad del e-learning o de la introducción de las aulas virtuales en las universidades. Nos centraremos únicamente en prácticas que han cobrado una especial fuerza en los últimos 5 o 10 años.

Uno de los conceptos más desafiantes para la educación superior en los últimos tiempos es el de Entorno Personal de Aprendizaje (PLE por sus siglas en inglés Personal Learning Environment). El PLE podría definirse como el entramado sociomaterial del que cada persona forma parte y usa de forma habitual para aprender, que ha existido siempre, pero que tiene especial sentido como espacio de reflexión y práctica (Castañeda y Adell, 2013). Desde este enfoque, que entiende que la educación formal es parte de un todo mayor que debería ser controlado y autorregulado por el individuo, se interpela directamente a algunos de los supuestos más tradicionales de las instituciones de educación superior exigiendo cambios (Castañeda, Dabbagh y Torres-Kompen, 2017).

Algunos de esos retos que propone el PLE tienen que ver con cambios en los roles de los participantes en los procesos de aprendizaje, con la organización de las enseñanzas, con la visión de las asignaturas como compartimentos no estancos y partes de un todo que, además, ya ni siquiera es completamente definido por la institución sino por el propio estudiante. Incluso plantea cambios en el perfil de la formación que se propone para los futuros profesionales (Prendes y Roman, 2017), 
quienes, al menos desde esta perspectiva, deberían aprender a enriquecer y gestionar su PLE y convertirse en aprendices autónomos y autorregulados (Barak, HusseinFarraj y Dori, 2016; Dabbagh y Kitsantas, 2012). Esto es algo que, según parece, no estamos consiguiendo.

Sin embargo, la otra cara de la moneda del aprendizaje a lo largo de la vida, la autorregulación y la emancipación, es que el sistema se desentienda de la formación de las personas al asumir que la responsabilidad y la "competencia para aprender" reside en el sujeto individual (Mulder, Weigel y Collings, 2008). Por consiguiente, la misma oferta educativa dependiente de la supuesta mirada personalizadora, se fragmenta cada vez más, y, lejos de asumir propuestas transdisciplinares o abiertas, propone "píldoras" de conocimiento "envasado" diseñadas para un consumo rápido.

En esta misma línea, metodologías docentes como las que se describen bajo el paraguas del término flipped classroom (o "clase invertida") han sido posibles gracias a la confluencia de dos condiciones tecnológicas recientes: la disponibilidad de repositorios de contenido multimedia, abiertos tanto para el almacenamiento como para el consumo (especialmente aquellos dedicados a la distribución de vídeo) y la universalización de dispositivos móviles conectados a Internet, desde los que se puede acceder a dichos contenidos en cualquier momento y lugar (Abeysekera y Dawson, 2015). Desde la "clase invertida", la acción del aula vuelve su mirada al trabajo en colaboración, al abandono de la tarima de la clase como púlpito unidireccional que los estudiantes solo observan de forma pasiva. El "discurso teórico" se cuela en los dispositivos del alumnado y se consume individualmente fuera del aula, para que, aprovechando la oportunidad de estar juntos "en" la clase, lleva a cabo dinámicas colaborativas de solución de problemas o debates cara a cara (Nouri, 2016; Strayer, 2012).

Pero, esta proliferación de vídeos cortos sobre temas específicos ha sido también una oportunidad para "trocear" el conocimiento, simplificarlo y "enlatarlo" como información, anulando casi por completo el uso de otras fuentes más adecuadas para propiciar análisis reflexivos o el desarrollo de competencias complejas, y no solo pasar pruebas de reconocimiento de conocimiento declarativo.

El uso del software social se ha materializado en las aulas en un aumento de las estrategias de enseñanza y aprendizaje activas, en las que los estudiantes crean un producto, lo cual, unido a la visibilidad de su trabajos en el universo en red, ha aumentado su nivel de participación y compromiso (engagement) (Schindler, Burkholder, Morad y Marsh, 2017). La razón quizá sea fruto del efecto novedad y del uso de códigos de comunicación más cercanos a los estudiantes de hoy. El resultado es un evidente aumento de la cantidad de trabajo de los estudiantes, pero que no siempre lleva aparejado un aumento de la reflexión y, por tanto, del aprendizaje.

La introducción de simulaciones, videojuegos -los llamados "juegos serios"- y de las mecánicas y dinámicas propias de los juegos en los contextos de educación superior (la "gamificación" del aprendizaje) ha aportado motivación e interés a los aprendizajes rutinarios. Según algunas revisiones de la investigación, con grandes 
resultados tanto a nivel cognitivo, como conductual y afectivo (Vlachopoulos y Makri, 2017). Ahora bien, la utilización de dinámicas de "gamificación" en las actividades de aprendizaje provoca también algunas dudas sobre los efectos secundarios. ¿¿ué supone estimular la competitividad entre iguales y el éxito como únicos valores? ¿Todo lo que el estudiante hace debe tener una respuesta inmediata y estimulante en forma de premio? ¿Aprenden que la motivación extrínseca, el premio, es la única deseable? ¿Cuánto tiempo de los estudiantes se dedica a la estrategia y hasta qué punto esta carga de trabajo extra deja a los estudiantes y a los profesores el tiempo necesario para la reflexión profunda sobre lo que se aprende?

La relación de la acción de aula con la tecnología digital se ha evidenciado también en los modelos de evaluación. Además de facilitar, y por tanto generalizar, la elaboración y uso de pruebas objetivas autocorrectivas de conocimiento declarativo, la tecnología también ha facilitado el uso de metodologías de evaluación como el portafolio o las rúbricas, que se han beneficiado de los formatos digitales para ampliar sus posibilidades expresivas y de reflexión (Tur y Marín, 2015). Otra práctica emergente, vinculada a las plataformas de e-learning, es el uso de analíticas del aprendizaje (learning analytics y social learning analytics) para proporcionar de modo automático retroalimentación personalizada que ayude a aumentar la calidad de la reflexión de los estudiantes (Buckingham Shum et al., 2016; Ferguson y Buckingham Shum, 2012). Sin embargo, aun teniendo esas herramientas, los niveles de reflexión sobre la acción entre los estudiantes son en demasiadas ocasiones excesivamente básicos (Llopis Nebot, 2017). En muchos casos, las rúbricas actúan como guías para completar y no como retos para desarrollarse, sirviendo como herramientas de hiperplanificación y control extremo que abundan en los ya conocidos mecanismos de performatividad (performativity) descritos por Ball (2003).

\section{Conflictos de lo digital en la investigación}

Todos los procesos relacionados con la investigación se han visto retados por el marco tecnológico digital. Science 2.o es el término genérico con el que se denominan las prácticas relativas a la "investigación aumentada" (Peña-López, 2013). Las relaciones investigador-tecnología han cambiado la forma misma de investigar (Castañeda, 2015). En el marco de una universidad neoliberal, orientada al mercado, competitiva y condicionada por rankings e indicadores, la tecnología ha cambiado también cómo se investiga y a los investigadores. Por ejemplo, cómo se hace una revisión de literatura contando con las bases de datos online o a través de sistemas de difusión de novedades (por ejemplo, Rich Site Summary o RSS) o la forma de proponer un problema y obtener feedback inmediato de redes de colegas alrededor del mundo (como Research Gate o Twitter). Se está pasando de trabajar con muestras a considerar la totalidad de la población (Big Data), se hacen análisis de datos contando con redes de ordenadores en todo el mundo, que de forma 
colaborativa nos ayudan con su fuerza de cálculo o se incorpora a los ciudadanos a la obtención de datos, la Citizen Science (Hand, 2010).

Pero al mismo tiempo todas estas posibilidades han complicado el aparataje teórico y estadístico de nuestras investigaciones hasta límites esperpénticos. Una complicación que, al menos en los resultados que denuncian diversos autores (Murphy y Costa, 2015, por ejemplo), va en detrimento de los procesos reflexivos y está aparejada a una alarmante escasez de la teorización, imprescindible para el avance de la ciencia, seguramente por falta de tiempo, de interés o de rentabilidad a la hora de convertirla en publicaciones punteras o financiación para proyectos de investigación. El Big Data amenaza con acabar con la teorización en las ciencias humanas: bastará que el software encuentre patrones entre los datos para, supuestamente, obtener "conocimiento".

Las posibilidades de "aumentación" de la investigación son particularmente llamativas en el ámbito de la difusión del conocimiento. La multiplicidad y variedad de códigos, formatos y canales de difusión, incluso la dificultad de poner barreras infranqueables a los canales digitales (o dicho de otro modo, la disponibilidad de puertas traseras en los muros de pago de las editoriales), han multiplicado la difusión de la investigación y ha facilitado que la comunicación abierta, democrática y pública (como, por otra parte, exigen cada vez más las instituciones públicas que financian investigación) sea cada día mayor (Fecher y Friesike, 2014; Veletsianos y Stewart, 2016).

Pero esa apertura y facilidad en la difusión también puede imprimir una alta dosis de marketing a la investigación, puede hacer que partamos de presupuestos más propios de los mercados que del objetivo universitario de la creación y difusión del conocimiento. Incluso produce relaciones complejas entre la realidad de la ciencia y la manera en que se transforma, difunde y propaga entre las personas, creando dinámicas problemáticas, entre la verdadera realidad de los investigadores y, por ejemplo, su repercusión en los medios o en los foros de intercambio (PlayerKoro, Bergviken Rensfeldt y Selwyn, 2017).

Las relaciones de identidad de los investigadores, cómo se relacionan con colegas e iguales a través de las redes sociales, cómo interactúan en términos de investigación y cómo consiguen el preciado objetivo de conocer e interactuar con investigación diferente de la suya, evidentemente ha cambiado. El "académico digital" (Costa, 2013; Veletsianos, 2016; Weller, 2011), entendido como aquel que discute, piensa y se relaciona con sus pares de todo el mundo y que hace crecer su perspectiva de investigación gracias al potencial de la tecnología digital, tiene sus propias perversiones.

Sin embargo, esa misma realidad presenta contradicciones que han convertido la vida académica en un mercado, una competición, una persecución sin fin de indicadores de impacto y ahora, además, pendiente de su imagen pública, de la galería de espejos que la refleja. Si bien hay un grupo de profesionales de la investigación que aprovecha estas oportunidades para enriquecer su perspectiva investigadora, en 
muchos casos -si quieren conservar su trabajo y posibilidades de promoción- tienen que mantener además intactas, si no aumentadas, las prácticas de investigación tradicionales. Son los double gamers (Costa, 2016), que entienden que este es el precio a pagar por sacar provecho de las bondades de la presencia online. Otros, en cambio, siguen la tendencia, pero usan la red simplemente como herramienta de marketing, como forma de promoción personal, el academic branding, o convierten las redes en una cámara de eco de sus propias ideas.

\section{Conflictos sobre el concepto de universidad digital}

A lo largo de la historia, las universidades han respondido a modelos diversos: el modelo francés o napoleónico, con instituciones públicas, dependientes de la administración y dedicadas fundamentalmente a la docencia; el modelo liberal anglosajón, centradoen el desarrollopersonal del estudiante; el modelo humboldtiano, basado en la libertad académica, imprescindible para la nueva ciencia experimental; y el modelo soviético, únicamente docente y en el que los ministerios ejercían la tutela directa de las titulaciones (Bricall, 1997). La idea de Universitas, entendida como el estudio universal, asociado a la libertad de pensamiento y de cátedra, así como la convivencia de profesores y estudiantes, ha marcado la personalidad de las universidades occidentales y -por el predominio político y económico de los países europeos- se extendió internacionalmente (Michavila y Calvo, 1999).

Ortega y Gasset (1930) afirmaba que la misión de la universidad era la generación, transmisión y crítica del conocimiento, la preparación para la vida profesional, el apoyo científico y técnico al desarrollo cultural, social y económico del entorno y la transmisión de la cultura universitaria.

En este sentido, la Carta Magna de las Universidades, firmada en 1988 en Bolonia, recuerda que las universidades son centro de cultura, de conocimiento y de investigación, y les corresponde transmitir los conocimientos, tanto a las nuevas generaciones como al conjunto de la sociedad. Una década después, en la Conferencia Mundial sobre la Educación Superior de 1998, UNESCO atribuye a la universidad la función principal de formar personas cultas, responsables y libres, que den sentido social a sus vidas. Es decir, no basta con formar profesionales con un conocimiento especializado, que únicamente se dediquen a sus propios intereses particulares, dejando de lado la comunidad, sino formar ciudadanos cultos, maduros y responsables ética y socialmente (De la Cruz, 2003; Martínez y Payà, 2007).

En las últimas décadas, la universidad ha crecido cuantitativamente de forma espectacular, pasando a ser una universidad de masas, se ha internacionalizado (o se le ha exigido que lo haga), ha convertido la docencia y la investigación en productos de mercado, compite en rankings por conseguir recursos y atraer a los estudiantes más capaces y, desde el poder político, se la espolea para que se convierta en un factor esencial en el crecimiento económico y competitividad global del país. Pero, al mismo tiempo, tras la crisis económica de la pasada década, a la universidad pública 
se le ha recortado la financiación en numerosos países. Además, ahora se enfrenta a nuevos retos relacionados con la revolución digital.

Como afirma Selwyn (2014), la tecnología es "un espacio de luchas complejas para controlar el corazón y el espíritu del sistema universitario público... un conjunto de problemas que se presentan como soluciones" (cap. 8, sec. Conclusions, para. 2). Los conflictos sobre tecnología educativa en la universidad no pueden entenderse sin referencia a debates más amplios sobre la función, estructura y métodos de la educación superior. Un debate sobre su futuro. La razón es doble: por una parte, el marco político de ideología neoliberal que impone, desde hace décadas, un modelo de universidad al servicio de la economía y, por otra, el hecho de que las tecnologías no son neutrales. Han sido diseñadas con unos objetivos determinados, se han seleccionado con criterios definidos, se despliegan de acuerdo a un plan y sus usuarios, profesores, estudiantes, etc., las utilizan desde expectativas y presupuestos sobre el conocimiento, la enseñanza y el aprendizaje universitarios determinados. Para complicar todavía más el marco de análisis de cualquier tecnología concreta, las herramientas tecnológicas se diseñan e implementan desde un "código técnico" ideológico, pero son ambivalentes (Feenberg, 2002; Hamilton y Feenberg, 2005), es decir, sus usuarios poseen cierta libertad para apropiárselas al ponerlas en acción.

Los análisis descontextualizados de las affordances de las herramientas, los típicos análisis de sus "posibilidades", sirven para dar ideas sobre su posible uso, pero es necesario situarlas en contextos concretos para comprender su impacto real en las actividades de enseñanza, aprendizaje y gestión universitarias. Las tecnologías educativas, además, se integran en estrategias de cambio más amplias, que tienen distintos significados para diferentes grupos de interés. Hamilton (2016) ha mostrado que el desarrollo de la educación online a lo largo de las últimas décadas debe entenderse como un espacio de lucha entre marcos de pensamiento rivales que compiten por imponer su visión sobre la naturaleza y objetivos de la universidad que entienden de manera diferente la enseñanza, el aprendizaje y el conocimiento que merece ser enseñado. Son visiones diferentes y contrapuestas del papel de la universidad en la sociedad. La hegemonía de la universidad neoliberal (Olssen y Peters, 2005; Roorda, 2013) frente a la universidad como servicio público (Corominas y Sacristán, 2010) o "comprometida" (Manzano, 2012) ha supuesto también el triunfo de una visión concreta de la universidad digital y de qué arquitectura tecnológica y herramientas son las más adecuadas para lograr sus fines (véase Laviña y Mengual (2008) como ejemplo de un modelo de la primera década del siglo XXI). La universidad actual se caracteriza por una visión instrumentalista de la tecnología, construida tanto a través del discurso como de las prácticas. Como denuncia Barnett (2016), la universidad se ha rendido a un nuevo lenguaje global, el del mundo empresarial, plagado de términos como empleabilidad, impacto, resultados, emprendimiento, competitividad, mercado, etc., "un discurso de la razón económica" (p. 18) al que le interesa estratégicamente que la tecnología sea percibida como neutra, transparente e inevitable. 


\section{EL FUTURO y algunas conclusiones abiertas}

La "neoliberalización tecnologizada" de la universidad ha multiplicado injustificadamente la cantidad de trabajo, la burocratización y el control. Ya no hay tiempo para el pensamiento crítico y el cuestionamiento del statu quo, uno de los objetivos de la educación superior. Un exceso de mecanismos de control al detalle de la actividad de docentes, estudiantes y personal que, lejos de aportar calidad o perspectiva, aumentan también la cantidad de trabajo y la percepción de que la evaluación es irrelevante. Dichos mecanismos han ampliado sin fin la necesidad de mantener un registro de todo lo que se hace, ignorando la razón por la que se hace y provocando un exceso de producción que no siempre redunda en un aumento de calidad. Se habla tranquilamente del "mercado de la educación superior", de marketing, de la necesaria reducción de costes, de economías de escala, etc. Todo ello se traduce en una actividad frenética para usar todos los canales y rellenar todas las opciones posibles de códigos y formatos, de actividades múltiples y variopintas gobernadas por una lógica gerencialista. Y para todo, las nuevas tecnologías digitales parecen ser las herramientas más adecuadas.

Por otra parte, si hay voluntad política, la tecnología también permite mayores niveles de personalización, participación y democratización y una auténtica apertura de la institución a la sociedad. Los investigadores, los docentes, los estudiantes y el personal están más y mejor "conectados" y disfrutan de un mayor potencial para crear y difundir un conocimiento más amplio, variado y complejo.

No obstante, la digitalización está afectando a todos los sectores productivos y ámbitos sociales en formas que nadie había previsto y que no se sabe a ciencia cierta hacia dónde nos llevarán ${ }^{1}$. Los cambios serán profundos y rápidos, y no solo en el ámbito del trabajo y la economía, sino también en el social y cultural. No pensemos que la universidad podrá permanecer ajena a ellos. ¿Cómo será la "universidad digital" del futuro? Quizá muy diferente a lo que imaginamos.

Algunos autores (Hall, 2016) ya nos han advertido cómo podría ser la universidad del futuro según el modelo de "capitalismo de plataforma" (Srnicek, 2016), tan de moda, de empresas innovadoras como Uber o Airbnb (Nurvala, 2015) como modelo organizativo de la universidad del futuro. Según Fitzgerald y Gunter (2017, pp. 257-8), "en muchos sentidos la neoliberalización ahora tiene una nueva cara" y ya hace tiempo que se detectan tendencias hacia la progresiva "uberificación" de la educación superior: clases "empaquetadas" en vídeo para consumir justin-time just-in-case, tutores bajo demanda, estandarización del currículum por competencias, plataformas de recomendación en función de perfiles, evaluación informatizada, etc. El Uberacadémico será un trabajador autónomo dedicado a preparar "paquetes de conocimiento" para instituciones-empresas diversas y a realizar video-tutorías online bajo demanda a través de plataformas que lo pondrán en contacto con sus clientes, los estudiantes interesados, y que gestionarán el pago. El conocimiento será accesible, fácil de consumir, just-in-time en función de las 
demandas del mercado. Los uberestudiantes podrían completar su currículum con objetos de aprendizaje estandarizados y homologados de diversas procedencias (en los MOOC de instituciones diversas, no necesariamente universitarias, por ejemplo), pasando una evaluación informatizada en la entidad que les otorga las credenciales (hecha por la empresa contratada al efecto) o reuniendo badges reconocidos por dicha institución. La Ubersidad no sería otra cosa que una marca comercial y una plataforma tecnológica que une oferta y demanda de formación y credenciales y que subcontrata a profesionales autónomos o empresas el resto de funciones. ¿Y la investigación? La harán las empresas, que saben cómo generar el único conocimiento valorado en nuestro tiempo: el útil para el sistema productivo.

La tecnología digital necesaria para este modelo de educación superior ya existe (“Uber-U is Already Here", 2016):

- Una aplicación móvil, que permite al usuario conectarse a un hub central de recomendación de contenidos de distintos "proveedores" y que resuelve las necesidades de orientación y tutorización "personalizadas" de los estudiantes conectándolos con los tutores disponibles, u otras formas de ayuda, de todo el mundo.

- Un sistema de seguimiento, que permite cobrar y transferir dinero (quizá en una criptomoneda) entre el alumno, la institución-empresa y los tutores autónomos.

- Una plataforma de evaluación estandarizada en línea, que permite, tras comprobar su identidad, verificar la competencia y las habilidades del estudiante de modo automatizado y/o con intervención humana.

- Una base de datos de cadena de bloques (blockchain) que mantiene un registro inalterable de cada transacción entre los participantes, para que la institución que otorga credenciales pueda verificar que el aprendizaje ha tenido lugar. Quizá con algunos smart contracts que emitan certificados de competencias sin intervención humana toda vez que se hayan cumplido ciertos requisitos (Bartolomé, Bellver, Castañeda y Adell, 2017).

Hemos dicho que el discurso sobre la universidad digital es un campo de batalla ideológico. Pero el conflicto debe generar propuestas. Y para poder entrar en ese campo es imprescindible no perder de vista tres características que influyen en la realidad que nos rodea y que deberían marcar un debate necesario sobre la universidad digital:

- Que el discurso sobre la "universidad digital”, siguiendo a Jasanoff (2015), es un imaginario socio-técnico, una visión de un futuro deseable que se mantiene colectivamente, se estabiliza institucionalmente y se ejecuta públicamente, y que está "animado por una comprensión compartida de formas de vida y de orden social alcanzables a través de, y apoyada en, avances en ciencia y tecnología" 
(p. 4). El discurso sobre la universidad digital es uno de los más importantes espacios de lucha ideológica.

- Que el paradigma de lo "digital” es cambiante. Aquello que nos parecía en sí misma una característica que definía claramente un contexto - digital- ha dejado de ser apelativo suficiente y ahora es una característica general que está marcando una época, pero que sigue evolucionando de formas impredecibles. Así, en educación superior no hablamos de lo mismo cuando nos referimos a los profundos cambios que supuso el e-learning para la teleenseñanza y la educación a distancia, cuando analizamos los cambios propiciados por la generalización del uso de tecnologías en las aulas y por parte de los profesores, cuando nos referimos a las implicaciones didácticas de la web 2.0 y los dispositivos personales, ni cuando pensamos en el tratamiento de grandes cantidades de datos, la inteligencia artificial, las cadenas de bloques, etc. "Digital" es un adjetivo que ya no describe casi nada en la universidad actual.

- Que la universidad digital es una realidad socio-material. No es la esencia inmutable de la tecnología digital la que influye en las universidades. Son las implementaciones de tecnologías digitales concretas, siempre al servicio de intereses de unos u otros grupos, lo que es necesario analizar al detalle. Es decir, la universidad es también un debate por definir las prácticas en la docencia, la investigación, la gestión y la difusión del conocimiento. $\mathrm{Y}$ es necesario considerar las redes de cada actante (personas, tecnologías, normas, etc.) que constantemente construyen y reconstruyen dichas prácticas, moduladas por la imprevisibilidad de algunos de los cambios que introducen las tecnologías. En último término, la tecnología la crean, desarrollan y despliegan seres humanos con intereses y necesidades concretas, y lo hacen de determinada manera y no otra. Por la misma razón, son susceptibles de ser subvertidas para promover prácticas más críticas, democráticas, abiertas y flexibles. La universidad digital es el producto de las complejas relaciones que se establecen entre tecnologías, personas y normas.

La universidad del presente ya es digital, aunque por el momento no ofrece nada sustancialmente diferente a la universidad neoliberal "analógica". Es una universidad que se acomoda dócilmente a las políticas hegemónicas, que tiene muy nítidas sus líneas de desarrollo y sus márgenes de actuación y que responde de forma inerte a los reclamos tecnológicos de su tiempo. Es más, creemos que todavía no existe ni siquiera el debate sobre cómo será la universidad del futuro, de un mundo en el que, si se cumplen las predicciones de algunos expertos, los robots y la inteligencia artificial eliminarán entre el 40 y el 50 por ciento del trabajo humano (Gunkel, 2017; Peters, 2017). ¿Cómo afrontará la universidad neoliberal la formación de personas para un mundo sin trabajo?

Poco o nada se ha dicho sobre cómo debe ser la tecnología en un modelo de universidad que responda a nuestro ideal de educación, a los fines de la sociedad en 
su conjunto y no del mercado, a la sociedad que queremos, no a la que se nos da hecha como inevitable. No depende solo de nosotros -es evidente-, pero de momento, si seguimos así, sin investigación, reflexión y debate, el modelo seguirá viniendo hecho y nosotros también seremos responsables.

\section{NOTAS}

1. Es tremendamente significativo que Ángel Gurría, Secretario General de la OCDE, titulara su reciente discurso ante el 2017 Global Strategy Group de la OCDE "Imprevisibilidad sin precedentes: transformación digital. El futuro del empleo y el comercio en un mundo digitalizado" (Gurría, 2017).

\section{REFERENCIAS BIBLIOGRÁFICAS}

Abeysekera, L., y Dawson, P. (2015). Motivation and cognitive load in the flipped classroom: definition, rationale and a call for research. Higher Education Research and Development, 34(1), 1-14. doi: https://doi.org/10.1080/07294360. 2014.934336

Area, M., San Nicolás, M., y Fariña, E. (2010). Buenas prácticas de aulas virtuales en la docencia universitaria semipresencial. Teoría de la Educación. Educación y Cultura en la Sociedad de la Información. 11(1). Recuperado de http://revistas. usal.es/index.php/revistatesi/article/ view/5787

Ball, S. J. (2003). The teacher's soul and the terrors of performativity. Journal of Education Policy, 18(2), 215-228. https://doi. org/10.1080/0268093022000043065

Barak, M., Hussein-Farraj, R., y Dori, Y. J. (2016). On-campus or online: examining self-regulation and cognitive transfer skills in different learning settings. International Journal of Educational Technology in Higher Education, 13(1), 35. doi: https://doi.org/10.1186/s41239016-0035-9

Barnett, R. (2016). Understanding the University: Institution, idea, possibilities. London; New York: Routledge.
Bartolomé, A. R., Bellver, C., Castañeda, L. J., y Adell, J. (2017). Blockchain en educación: introducción y crítica al estado de la cuestión. Edutec. Revista Electrónica de Tecnología Educativa, 61, 1-14. doi: https://doi.org/10.21556 edutec.2017.61.915

Bricall, J. M. (1997). Visión de la Universidad en Europa: problemas diversos, soluciones comunes. En Los nuevos desafíos de la Universidad. Madrid: Institución Libre de Enseñanza.

Buckingham Shum, S., Sándor, Á., Goldsmith, R., Wang, X., Bass, R., y McWilliams, M. (2016). Reflecting on reflective writing analytics: Assessment challenges and iterative evaluation of a prototype tool. En Proceedings of the Sixth International Conference on Learning Analytics \& Knowledge (pp. 213-222). New York, NY, USA: ACM. doi: https:// doi.org/10.1145/2883851.2883955

Cabero, J. (2005). Las TIC y las universidades: retos, posibilidades $\mathrm{y}$ preocupaciones. Revista de la Educación Superior, XXXIV(3), 135. Recuperado de http://www.redalyc.org/resumen. oa?id=60413505

Castañeda, L. (2015). Investigación, conocimiento y participación en la academia 2.0: de la torre de marfil a la 
galería de los espejos. Temps d'Educació, (49), 89-104. Recuperado de http://www. raco.cat/index.php/TempsEducacio/ article/view/303524

Castañeda, L., y Adell, J. (2013). Entornos personales de aprendizaje: claves para el ecosistema educativo en red. Alcov: Editorial Marfil. Recuperado de http:/ www.um.es/ple/libro/

Castañeda, L., Dabbagh, N., y TorresKompen, R. (2017). Personal learning environments: Research-Based practices, frameworks and challenges. Journal of New Approaches in Educational Research, 6(1), 1-2. doi: https://doi. org/10.7821/naer.2017.1.229

Corominas, A. y Sacristán, V. (2010). Construir el futuro de la universidad pública. Barcelona: Icaria editorial.

Costa, C. (2013). The habitus of digital scholars. Research in Learning Technology, 21(1), 21274. doi: https:/ doi.org/10.3402/rlt.v21.21274

Costa, C. (2016). Double gamers: academics between fields. British Journal of Sociology of Education, 37(7), 993-1013. doi: https://doi.org/10.1080/01425692.2 014.982861

Dabbagh, N., y Kitsantas, A. (2012). Personal learning environments, social media, and self-regulated learning: A natural formula for connecting formal and informal learning. The Internet and Hiqher Education, 15(1), 3-8. doi: https://doi. org/10.1016/j.iheduc.2011.06.002

De la Cruz, M. Á. (2003). Necesidad y objetivos de la formación pedagógica del profesor universitario. Revista de Educación, 331, 35-66. Recuperado de https://sede.educacion.gob.es publiventa/detalle.action?cod=11294

Dunleavy, P., Margetts, H., Bastow, S., y Tinkler, J. (2006). New Public Management Is Dead -Long Live Digital-Era Governance. Journal of Public Administration Research and
Theory, 16(3), 467-494. doi: https://doi. org/10.1093/jopart/muio57

Fecher, B., y Friesike, S. (2014). Open science: one term, five schools of thought. En S. Bartling y S. Friesike (Eds.), Opening science. The evolving guide on how the Internet is changing research, collaboration and scholarly publishing (17-47). Cham: Springer. doi: https://doi. org/10.1007/978-3-319-00026-8 2

Feenberg, A. (2002). Transforming technology. Oxford University Press.

Fenwick, T., y Edwards, R. (2017). How sociomaterial approaches could support higher education as a critical practice. En B. Leibowitz, V. Bozalek, y P. Kahn (Eds.), Theorising learning to teach in higher education (34-52). London; New York: Routledge. Recuperado de https://www.tavlorfrancis com/books/e/9781317195733 chapters/10.4324\%2F9781315559605-10

Ferguson, R., y Buckingham Shum, S. (2012). Social learning analytics: five approaches. Presentado en 2nd International Conference on Learning Analytics \& Knowledge, Vancouver, British Columbia, Canada. (pp. 23-33) doi: https://doi. org/10.1145/2330601.2330616

Fitzgerald, T., y Gunter, H. M. (2017). Debating the agenda: the incremental uberisation of the field. Journal of Educational Administration and History, 49(4), 257-263. doi:https://doi.org/10.10 80/00220620.2017.1342980

Floridi, L. (2017). The fourth revolution: how the Infosphere Is Reshaping Human Reality. Oxford: Oxford University Press.

Gunkel, D. J. (2017). Rage Against the Machine: Rethinking Education in the Face of Technological Unemployment. En K. LaGrandeur y J. J. Hughes (Eds.), Surviving the machine age (147-162). Cham: Springer. doi: https://doi. org/10.1007/978-3-319-51165-8_10

Gurría, A. (2017, Noviembre 24). Unprecedented unpredictability: digital 
transformation. The future of jobs and trade in a digitalised world. Recuperado de http://www.oecd.org/employment gsg-future-of-jobs-in-digitalised-worldnov-2017.htm

Hall, G. (2016). The Uberfication of the University. Minnesota, USA: University of Minnesota Press.

Hamilton, E. C. (2016). Technology and the politics of university reform: the social shaping of online education. New York: Palmgrave Macmillan.

Hamilton, E., y Feenberg, A. (2005). The technical codes of online education. E-learning and Diqital Media, 2(2), 104-121. doi: https://doi.org/10.2304 elea.2005.2.2.1

Hand, E. (2010). Citizen science: People power. Nature News, 466(7307), 685-687. doi: https://doi.org/10.1038/466685a

Jasanoff, S. (2015). Future imperfect: science, technology, and the imaginations of modernity. En S. Jasanoff y S.-H. Kim (Eds.), Dreamscapes of modernity: sociotechnical imaginaries and the fabrication of power. Chicago, IL: University of Chicago Press.

Laviña, J., y Mengual, L. (Eds.). (2008). Libro blanco de la universidad digital 2010. Madrid: Ariel-Fundación Telefónica. Recuperado de https://www. fundaciontelefonica.com/arte cultura/ publicaciones-listado/pagina-itempublicaciones/itempubli/22

Llopis Nebot, A. M. (2017, Septiembre 21). Pensamiento reflexivo en el Prácticum I del alumnado de Grado de Maestro en Educación Primaria a través de diarios online (Ph.D. Thesis). Universitat Jaume I. Recuperado de http://hdl.handle. net/10803/430854

Manzano Arrondo, V. (2012). La universidad comprometida. Bilbao: Universidad del País Vasco-Euskal Herriko Unibertsitatea, Hegoa. Recuperado de http:/ publicaciones.hegoa.ehu.es/uploads pdfs/212/Univ comprometida.pdf
Martínez, F., y Prendes, M. P. (2003). ¿Adónde va la educación en un mundo de tecnologías? En F. Martínez (Ed.), Redes de comunicación en la enseñanza (281300). Barcelona: Paidós. Recuperado de https://dialnet.unirioja.es/servlet/ articulo?codigo=756021

Martínez, M., y Payà, M. (2007). La formación de la ciudadanía en el Espacio Europeo de Educación Superior. En J. L. García Garrido (Ed.), Formar ciudadanos europeos. Madrid: Academia Europea de Ciencias y Artes.

Michavila, F., y Calvo, B. (1999). La Universidad española hacia Europa. Madrid: Fundación Alfonso Martín Escudero.

Mulder, M., Weigel, T., y Collings, K. (2008). El concepto de competencia en el desarrollo de la educación y formación profesional en algunos Estados miembros de la UE: un análisis crítico. Profesorado: Revista de Curriculum y Formación del Profesorado, 12(3), 7. Recuperado de https://www.ugr.es/ recfpro/ rev123ART6.pdf

Murphy, M., y Costa, C. (Eds.). (2015). Theory as method in research: on Bourdieu, social theory and education. London; New York, NY: Routledge.

Nouri, J. (2016). The flipped classroom: for active, effective and increased learning especially for low achievers. International Journal of Educational Technology in Higher Education, 13, 33. doi: https:// doi.org/10.1186/s41239-016-0032--

Nurvala, J.-P. (2015). 'Uberisation' is the future of the digitalised labour market. European View, 14(2), 231-239. doi: https://doi.org/10.1007/s12290-015 0378-y

Olssen, M., y Peters, M. A. (2005). Neoliberalism, higher education and the knowledge economy: from the free market to knowledge capitalism. Journal of Education Policy, 
2O(3), 313-345. doi: https://doi. org/10.1080/02680930500108718

Ortega y Gasset, J. (1930). Misión de la universidad. Madrid: Alianza Editorial.

Pardo, A., Jovanovic, J., Dawson, S., Gašević, D., y Mirriahi, N. (2017). Using learning analytics to scale the provision of personalised feedback: Learning analytics to scale personalised feedback. British Journal of Educational Technology. doi: https://doi.org/10.1111/bjet.12592

Peña-López, I. (2013). El PLE de investigación-docencia: el aprendizaje como enseñanza. En L. Castañeda y J. Adell (Eds.), Entornos personales de aprendizaje: claves para el ecosistema educativo en red (93-110). Alcov: Marfil. Recuperado de https://digitum.um.es xmlui/bitstream/10201/30413/1/ capitulo6.pdf

Peters, M. A. (2017). Technological unemployment: educating for the fourth industrial revolution. Journal of Self-Governance and Management Economics, 5(1), 25. doi: https://doi. org/10.22381/JSME5120172

Player-Koro, C., Bergviken Rensfeldt, A., y Selwyn, N. (2017). Selling tech to teachers: education trade shows as policy events. Journal of Education Policy, 1-22. doi: https://doi.org/10.1080/02680939. 2017.1380232

Prendes, M. P., y Roman, M. (2017). Entornos personales de aprendizaje: Una visión actual de cómo aprender con tecnologías. Barcelona: Octaedro.

Roorda, S. T. (2013). Towards the 'neoliberal' university. Higher education and innovation in the knowledge economy (Doctoral dissertation). Maastricht University, Maastricht. Recuperado de https://www.academia.edu/9161870/ Towards the Neoliberal University Higher Education and Innovation in the Knowledge Economy

Salinas, J. (1999). Enseñanza flexible, aprendizaje abierto. Las redes como herramienta para la formación. Edutec. Revista Electrónica de Tecnología Educativa, (10). doi: https://doi. org/10.21556/edutec.1999.10.567

Schindler, L. A., Burkholder, G. J., Morad, O. A., y Marsh, C. (2017). Computer-based technology and student engagement: a critical review of the literature. International Journal of Educational Technology in Higher Education, 14, 25. doi: https://doi.org/10.1186/s41239-0170063-0

Selwyn, N. (2014). Digital technology and the contemporary university: degrees of digitization. London: Society for Research into Higher Education. Recuperado de https://www.amazon.com/DigitalTechnology-Contemporary-Universitydigitization-ebook/dp/BooKIRAZ4G/

Srnicek, N. (2016). Platform Capitalism. Cambridge, UK ; Malden, MA: Theory Redux. Recuperado de https://www.wiley.com/en-us Platform+Capitalism-p-9781509504909

Strayer, J. F. (2012). How learning in an inverted classroom influences cooperation, innovation and task orientation. Learning Environments Research, 15(2), 171-193. doi: https://doi. org/10.1007/s10984-012-9108-4

Tur, G., y Marín, V. (2015). Exploring students' attitudes and beliefs towards e-portfolios and technology in education. Enseñanza \& Teaching, 33(1), 57-82. doi: https://doi.org/10.14201/et20153315782

Uber-U is already here. (6 de mayo de 2016). Recuperado de https://teachonline.ca tools-trends/exploring-future-education uber-u-already-here

Veletsianos, G. (2016). Social media in academia: networked scholars. Nueva York: Routledge.

Veletsianos, G., y Stewart, B. (2016). Discreet openness: scholars' selective and intentional self-disclosures online. Social Media + Society, 2(3). doi: https://doi. org/10.1177/2056305116664222 
Vlachopoulos, D., y Makri, A. (2017). The effect of games and simulations on higher education: a systematic literature review. International Journal of Educational Technoloqu in Hiqher Education, 14, 22. doi: https://doi.org/10.1186/s41239-0170062-1
Weller, M. (2011). The digital scholar: how technology is transforming academic practice. London: Bloomsbury Academic. Recuperado de https:/ www.bloomsbury.com/uk/the-digitalscholar-9781849666268

\section{PERFIL ACADÉMICO Y PROFESIONAL DE LOS AUTORES}

Jordi Adell Segura. Doctor en Filosofía y Ciencias de la Educación y profesor titular del Depto. de Educación de la Universitat Jaume I (Castellón, España). Investigador principal del grupo de investigación GREAT (Enseñanza, Aprendizaje y Tecnología) y del Centro de Educación y Nuevas Tecnologías (CENT) de la Universitat Jaume I. Sus líneas de investigación actuales incluyen el análisis y el desarrollo de la competencia digital en la formación inicial docente y el impacto de la tecnología en la enseñanza y el aprendizaje universitarios.

E-mail: iordi@uji.es

\section{DIRECCIÓN DEL AUTOR}

Dept. d'Educació

Universitat Jaume I

Avda. Sos Baynat, s/n.

12071 Castelló de la Plana (España)

Linda Castañeda Quintero. Doctora en Tecnología Educativa por la Universitat de les Illes Balears. Profesora contratada doctora del Departamento de Didáctica y Organización Escolar de la Universidad de Murcia y miembro del Grupo de Investigación de Tecnología Educativa (GITE) de la misma universidad. Su investigación se interesa especialmente por la implementación de pedagogías emergentes en contextos formales y no formales, Entornos personales de Aprendizaje (PLE) y factores clave de desarrollo curricular en el mundo digital.

E-mail: lindacq@um.es

\section{DIRECCIÓN DE LA AUTORA}

Facultad de Educación

Universidad de Murcia

30100 Murcia (España) 
Francesc Esteve Mon. Profesor Ayudante Doctor del Departamento de Educación de la Universitat Jaume I (Castellón, España). Miembro del grupo de investigación GREAT (Enseñanza, Aprendizaje y Tecnología) de la Universitat Jaume I. Colaborador del grupo de investigación ARGET (Applied Research Group in Education and Technology) y de la Cátedra UNESCO de Gestión y Política Universitaria. Centra su investigación en el análisis de la competencia digital y la formación inicial docente.

E-mail: festeve@uji.es

\section{DIRECCIÓN DEL AUTOR}

Facultat de Ciències Humanes i Socials, Universitat Jaume I

Avda. Sos Baynat s/n.

12071 Castelló de la Plana (España)

Fecha de recepción del artículo: 15/12/2017

Fecha de aceptación del artículo: 17/01/2018

\section{Como citar este artículo:}

Adell Segura, J., Castañeda Quintero, L., y Esteve Mon, F. (2018). Hacia la Ubersidad? Conflictos y contradicciones de la universidad digital. RIED. Revista Iberoamericana de Educación a Distancia, 21(2), pp. 51-68. doi: http://dx.doi. org/10.5944/ried.21.2.20660 